This item was submitted to Loughborough's Research Repository by the author.

Items in Figshare are protected by copyright, with all rights reserved, unless otherwise indicated.

\title{
Game theoretic data association for multi-target tracking with varying number of targets
}

PLEASE CITE THE PUBLISHED VERSION

https://doi.org/10.1109/RADAR.2016.7485219

PUBLISHER

(C) IEEE

VERSION

AM (Accepted Manuscript)

LICENCE

CC BY-NC-ND 4.0

\section{REPOSITORY RECORD}

Daniyan, Abdullahi, Yu Gong, and Sangarapillai Lambotharan. 2019. "Game Theoretic Data Association for Multi-target Tracking with Varying Number of Targets”. figshare. https://hdl.handle.net/2134/37347. 


\title{
Game Theoretic Data Association for Multi-target Tracking with Varying Number of Targets
}

\author{
Abdullahi Daniyan*, Yu Gong, and Sangarapillai Lambotharan
}

\begin{abstract}
We investigate a game theoretic data association technique for multi-target tracking (MTT) with varying number of targets. The problem of target state-estimate-to-track data association has been considered. We use the SMC-PHD filter to handle the MTT aspect and obtain target state estimates. We model the interaction between target tracks as a game by considering them as players and the set of target state estimates as strategies. Utility functions for the players are defined and a regret-based learning algorithm with a forgetting factor is used to find the equilibrium of the game. Simulation results are presented to demonstrate the performance of the proposed technique.
\end{abstract}

Index Terms-Multi-target tracking (MTT), data association, game theory, correlated-equilibrium, forgetting factor, regret matching, particle filter, sequential Monte Carlo (SMC), probability hypothesis density (PHD) filter.

\section{INTRODUCTION}

In multi-target tracking (MTT) problems, the objective is to jointly estimate target states and number from a set of corrupted observations [1]. Furthermore, not all observations/measurements received by sensors at each time instance are from existing targets. The sensor may pick up spurious detections due to clutter or may miss detections. As a result, the measurements received at each time step are corrupted and consist of indistinguishable measurements that may be either targetoriginated or due to clutter. It is therefore crucial in MTT to ascertain which measurement is due to which target. Data association deals with the problem of assigning measurements obtained to individual targets [2].

In solving the MTT problem, the most common methods are: i) those that apply explicit data association techniques to assign measurements to each track [3], and ii) those that try to avoid explicit association techniques [1]. In the first case, data association techniques are used to assign measurements to each track and a single target tracker [4]-[6] (for example the Kalman filter (KF) or the particle filter (PF)) is used for that track. In this setting, the number of targets needs to be known and fixed. Several techniques are used to achieve data association. The most common data association techniques include the nearest neighbour (NN) [4], the multiple hypothesis tracker (MHT) [4], and the joint probabilistic data association (JPDA) filter [4], [7]. In NN, at each time step, the predicted target state is associated with the closest measurement. MHT however keeps track of, and carries forward, all the association hypotheses to the next time step and aggregates them over time. The JPDA finds the association probabilities during each time update by considering all the targets and the measurements simultaneously and merging many hypotheses to form a single track hypothesis following a validation process. However, NN, MHT and JPDA algorithms are more suited to linear update/linear measurement and Gaussian uncertainty scenarios [8]. Recently, [8] and [9] proposed a measurement-to-track data association for MTT using game theory. In their approach, a known and fixed number of targets was considered and the aim was to use game theory to assign measurements to individual single target trackers. Based on the number of players, the individual trackers were set and the interaction between the trackers was formulated as a game.

A. Daniyan (*corresponding author), Y. Gong and S. Lambotharan are with the School of Electronic, Electrical and Systems Engineering, Loughborough University, U.K. (\{a.daniyan, y.gong, s.lambotharan\}@lboro.ac.uk)
A regret based algorithm was used to find the equilibrium of the game. This approach is however constrained because the number of targets needs to be known and fixed, and the regret of all players is accumulated and kept.

Those data association techniques that avoid explicit associations between measurements and tracks include the sparse-grid quadrature non-linear filter [10], multi-target particle filters [11] and the random finite set (RFS) method [5], [6], [12]. The modelling of target states and observations as an RFS allows the use of the Bayesian filtering approach [13] to estimate the multi-target states in the presence of clutter, missed detections and association uncertainty [14]. The RFS based probability hypothesis density (PHD) filter [5], [6] and the multi-target multi-Bernoulli (MeMBer) filter are two most tractable alternatives to the optimal multi-target filter. [5], [6], [15] and [16]. The PHD filter is a recursion that propagates the first order moment of the RFS of targets in time [6]. The PHD filter is able to track time varying multi-targets without the need to explicitly associate measurements to tracks. The PHD filter has been implemented in two distinct fashions; i.e. as the Gaussian mixture PHD (GM-PHD) filter [13] and the sequential Monte Carlo PHD (SMC-PHD) filter [17]. In the SMC-PHD filter implementation, the PHD is approximated by a set of weighted particles and therefore more suitable for tracking in non-linear and non-Gaussian environments.

In this paper, we propose a game theoretic approach to solve the data association problem for varying number of targets in MTT. This approach involves firstly, using the SMC-PHD filter to track targets utilizing all available measurements to obtain target state estimates. Then our proposed game theoretic method is used to perform target state estimate-to-track data association. The key differences between our approach and [8], [9] are: i) we consider a varying number of targets by using the SMC-PHD filter, ii) we use a forgetting factor to avoid accumulating and keeping the regrets of each player. The use of a game theoretic approach allows for data association, for non-linear, non-Gaussian scenarios. Also, using game theory, data association is simultaneous rather than sequential as opposed to other data association algorithms [8], [9].

To find target state-estimate-to-track associations, we formulate the problem of data association as a game between multiple and varying number of tracks. The strategies and utility function of each track are specified. A regret-based learning algorithm with a forgetting factor or memory is then used to find the equilibrium of this game. Correlated equilibrium is used as a stable operating point. Also, the set of correlated equilibria is a generalization of Nash equilibria and correlated equilibria are more preferable than Nash equilibria since they directly consider the ability of agents to coordinate their actions and this coordination leads to better performance [18].

\section{PRoblem Formulation}

This section presents the MTT problem for varying number of targets. We first describe the multi-target state and measurement models and then describe how target state estimates are obtained using the SMC-PHD filter. 


\section{A. State Model}

Let the non-linear state evolution model of a target be:

$$
\mathbf{x}_{k}=\mathbf{f}\left(\mathbf{x}_{k-1}, \mathbf{v}_{k}\right)
$$

where $\mathbf{x}_{k}$ denotes target state at discrete time $k, \mathbf{v}_{k}$ is an independent and identically distributed (i.i.d.) process noise vector and $\mathbf{f}(\cdot)$ is the non-linear system transition function. Then the multi-target state at time $k$ can be written as $\boldsymbol{X}_{k}=\left\{\mathbf{x}_{1, k}, \ldots, \mathbf{x}_{M, k}\right\} \in E_{s}$ where $M$ is the number of targets present at time $k$, and $E_{s}$ denotes the state space.

\section{B. Measurement Model}

The target-originated non-linear measurement model is given as:

$$
\mathbf{z}_{k}=\mathbf{h}_{k}\left(\mathbf{x}_{k}, \mathbf{n}_{k}\right)
$$

where $\mathbf{h}_{k}(\cdot)$ is a non-linear function, and $\mathbf{n}_{k}$ is an i.i.d. process noise vector. Measurements consist of both target-originated measurements and false alarms. The multi-target measurement set at time $k$ in the observation space is: $\boldsymbol{Z}_{k}=\left\{\mathbf{z}_{1, k}, \ldots, \mathbf{z}_{a, k}\right\} \bigcup\left\{\mathbf{c}_{1, k}, \ldots \mathbf{c}_{b, k}\right\} \in E_{o}$ where $a$ denotes the number of target-originated measurements, $b$ denotes the number of false measurements and $E_{o}$ denotes the observation space. Then the multi-target cumulative measurement set at time $K$ is $\boldsymbol{Z}_{1: K}=\left\{\mathbf{Z}_{1}, \mathbf{Z}_{2}, \ldots, \mathbf{Z}_{K}\right\} \in E_{o}$.

\section{MTT using SMC-PHD filter}

1) Initialization: In the SMC-PHD filter proposed in [19], [20], at time $k=1$ the PHD $D_{k \mid k}$ is represented by a number of particles with associated weights $\left\{\mathbf{x}_{k}^{i}, w_{k}^{i}\right\}_{i=1}^{L_{k}} . L_{k}$ is the number of all surviving particles at time $k$. A particle approximation of the intensity function at time step, $k>1$, can be obtained from a particle distribution at the previous time step using prediction and update stages.

2) Prediction: Apply importance sampling to generate $L_{k-1}$ and $J_{k}$ particles from two proposal densities $\left(q_{k}(\cdot \mid \cdot)\right.$ and $\left.p_{k}(\cdot \mid \cdot)\right)$ to represent persistent and new born targets with associated weights respectively, with $\mathcal{L}_{k}=L_{k-1}+J_{k}$ i.e.

$$
\begin{gathered}
\tilde{\mathbf{x}}_{k \mid k-1}^{i} \approx \begin{cases}q_{k}\left(\cdot \mid \tilde{\mathbf{x}}_{k-1}^{i}, \mathbf{Z}_{k}\right), & i=1, \ldots, L_{k-1} \\
p_{k}\left(\cdot \mid \mathbf{Z}_{k}\right), & i=L_{k-1}+1, \ldots, \mathcal{L}_{k}\end{cases} \\
\tilde{w}_{k \mid k-1}^{i}= \begin{cases}\frac{\phi_{k \mid k-1}\left(\tilde{\mathbf{x}}_{k}^{i}, \tilde{\mathbf{x}}_{k-1}^{i}\right)}{q_{k}\left(\tilde{\mathbf{x}}_{k \mid k-1} \mid \tilde{\mathbf{x}}_{k-1}^{i}, \mathbf{Z}_{k}\right)} w_{k-1}^{i}, & i=1, \ldots, L_{k-1} \\
\frac{\gamma_{k}\left(\tilde{\mathbf{x}}_{k}^{i}\right)}{J_{k} p_{k}\left(\tilde{\mathbf{x}}_{k \mid k-1} \mid \mathbf{Z}_{k}\right)}, & i=L_{k-1}+1, \ldots, \mathcal{L}_{k}\end{cases}
\end{gathered}
$$

where

$\phi_{k \mid k-1}\left(\tilde{\mathbf{x}}_{k}, \tilde{\mathbf{x}}_{k-1}\right)=p_{S}\left(\tilde{\mathbf{x}}_{k-1}\right) f_{k \mid k-1}\left(\tilde{\mathbf{x}}_{k}, \tilde{\mathbf{x}}_{k-1}\right)+b_{k \mid k-1}\left(\tilde{\mathbf{x}}_{k}, \tilde{\mathbf{x}}_{k-1}\right)$, $\gamma_{k}(\cdot)$ is the PHD of the spontaneous birth, $p_{S}(\cdot)$ is the probability of the target survival, $f_{k \mid k-1}\left(\tilde{\mathbf{x}}_{k}, \tilde{\mathbf{x}}_{k-1}\right)$ is the single target motion model, and $b_{k \mid k-1}\left(\tilde{\mathbf{x}}_{k}, \tilde{\mathbf{x}}_{k-1}\right)$ is the PHD of spawned targets.

3) Update: For each $\mathbf{z} \in \mathbf{Z}_{k}$, compute:

$$
C_{k}(\mathbf{z})=\sum_{i=1}^{\mathcal{L}_{k}} \psi_{k, \mathbf{z}}^{i}\left(\tilde{\mathbf{x}}_{k}^{i}\right) \tilde{w}_{k \mid k-1}^{i},
$$

where $\psi_{k, \mathbf{z}}\left(\tilde{\mathbf{x}}_{k}\right)=p_{D}\left(\tilde{\mathbf{x}}_{k}\right) g\left(\mathbf{z} \mid \tilde{\mathbf{x}}_{k}\right), p_{D}\left(\tilde{\mathbf{x}}_{k}\right)$ is the probability of detection, and $g\left(\mathbf{z} \mid \tilde{\mathbf{x}}_{k}\right)$ is the measurement likelihood function for the single target.

Then, for $i=1, \ldots, \mathcal{L}_{k}$, update the weights using:

$$
\tilde{w}_{k}^{i}=\left[\nu\left(\tilde{\mathbf{x}}_{k}^{i}\right)+\sum_{\mathbf{z} \in \mathbf{Z}_{k}} \frac{\psi_{k, \mathbf{z}}^{i}\left(\tilde{\mathbf{x}}_{k}^{i}\right)}{\kappa_{k}(\mathbf{z})+C_{k}(\mathbf{z})}\right] \tilde{w}_{k \mid k-1}^{i}
$$

where $\nu\left(\tilde{\mathbf{x}}_{k}\right)=1-p_{D}\left(\tilde{\mathbf{x}}_{k}\right)$ is the probability of target non-detection, and $\kappa_{k}(\mathbf{z})=\lambda_{k} c_{k}(\mathbf{z})$ is the clutter intensity, $\lambda_{k}$ is the average number of Poisson clutter points per scan, and $c_{k}(\mathbf{z})$ is the probability density over the state-space of the clutter point.
4) Resample and Clustering: The number of targets at time $k$ is computed as $T_{k}=\operatorname{round}\left(\sum_{i=1}^{\mathcal{L}_{k}} \tilde{w}_{k}^{i}\right) . L_{k}=\rho T_{k}$ particles are resampled to avoid degeneracy. Clustering is performed on resampled particles to obtain the target state estimates, $\tilde{\boldsymbol{x}}_{k}$.

Therefore at time $k$, the output of the SMC-PHD filter is a set of target state estimates given as: $\tilde{\boldsymbol{x}}_{k}=\left\{\tilde{\boldsymbol{x}}_{1, k}, \tilde{\boldsymbol{x}}_{2, k}, \cdots, \tilde{\boldsymbol{x}}_{T_{k}, k}\right\}$, where $\tilde{\boldsymbol{x}}_{t, k}=\left[x_{t, k}, \dot{x}_{t, k}, y_{t, k}, \dot{y}_{t, k}\right]^{\prime}, x_{t, k}$ and $y_{t, k}$ are the $\mathrm{x}$ and $\mathrm{y}$ positions at time $k$ and $\dot{x}_{t, k}$ and $\dot{y}_{t, k}$ are the velocity in $\mathrm{x}$ and $\mathrm{y}$ directions for the $t$ th target respectively. It is the assumption that, each target, when present generates at most one measurement. Also, most false alarms have been filtered out during the SMC-PHD filtering. This implies that the output of the SMC-PHD filter are target state estimates only with no false alarm. In the case where a false alarm was not filtered, it will be recorded as a new target. Figure 1 shows the different stages

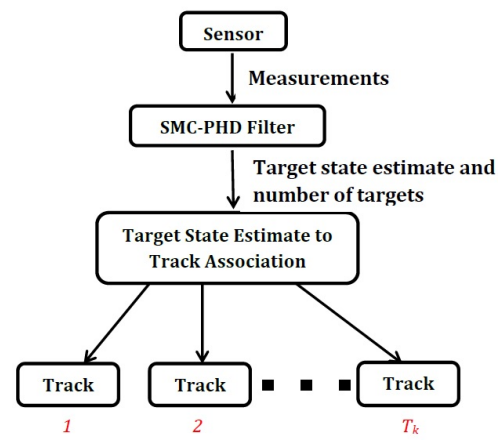

Figure 1: Block diagram showing various stages of the data association process.

in our proposed approach. Both target-originated measurements and clutter are obtained from a sensor. The SMC-PHD filter tracks the targets using these measurements while filtering out clutter to obtain target state estimates. The proposed game theoretic approach is then used to associate these target state estimates to various target tracks.

\section{DATA ASSOCIATION FOR VARYING NUMBER OF TARGETS USING GAME THEORY}

In this section, we describe how the game between the different tracks is played at each iteration of the SMC-PHD filter output.

\section{A. The Game}

Consider a game at time $k$ with a set, $\Omega_{k}$ of $\mathcal{P}_{k}$ players. The players, $\mathcal{P}_{k}$ are the tracks for different targets and can vary depending on the estimated number of targets by the SMC-PHD filter, $T_{k}$. Each player wants to assign a new target state estimate to its existing track. We say that a new player has joined the game at time $k$ when $T_{k}>$ $T_{k-1}$ and a player has left when $T_{k}<T_{k-1}$. The strategy set $\mathcal{S}_{p, k}=$ $\left\{0,1,2, \cdots, T_{k}\right\}$ of each player corresponds to the set of target state estimates from the SMC-PHD filter and they are known to all of the players at time $k$. The strategy $s_{p, k} \in \mathcal{S}_{p, k}$ allows the player to choose one target state estimate from the set of all target state estimates or 0 in the case that the corresponding target does not produce any tracks or disappears from the tracking scene. $\mathcal{P}_{k}$ is the number of players at time $k$ and corresponds to $T_{k}$. For each player, a utility function is defined as $u_{p, k}\left(s_{p, k}, s_{-p, k}\right): \mathcal{S} \longrightarrow \mathbb{R}$, with $\mathcal{S}=\mathcal{S}_{1} \times \cdots \times \mathcal{S}_{\mathcal{P}, k}$ where $-p_{k}$ refers to all players except player $p_{k}$. $\mathcal{U}_{k}$ denotes the set of utility functions of all the players, i.e., $\mathcal{U}_{k}=\left\{u_{p_{k}}\right\}_{p_{k}=1}^{\mathcal{P}_{k}}$. The game $\Gamma_{k}\left(\Omega_{k}, \mathcal{S}_{k}, \mathcal{U}_{k}\right)$ defined by the set of players $\Omega_{k}$, the strategy set $\mathcal{S}_{k}$, and the utility functions $\mathcal{U}_{k}$ is a one shot game played at the end of each iteration of the MTT tracker and is called the MTT data association game for varying number of targets. 


\section{B. Utility Functions}

For the $p$ th player at time $k$, the utility function similar to [8] is defined as follows:

$u_{p, k}\left(s_{p, k}, s_{-p, k}\right)= \begin{cases}d_{p, k}\left(s_{p, k}\right)+\mu_{1} g_{p, k}\left(s_{p, k}, s_{-p, k}\right), & s_{p, k} \neq 0 \\ \mu_{2}, & s_{p, k}=0\end{cases}$

where

$$
d_{p, k}\left(s_{p, k}\right)=-\left[\left(\boldsymbol{x}_{s_{p}, k}-\tilde{\boldsymbol{x}}_{p, k}\right)^{T} \boldsymbol{\Sigma}^{-1}\left(\boldsymbol{x}_{s_{p}, k}-\tilde{\boldsymbol{x}}_{p, k}\right)-d_{\max }\right]
$$

is the scaled Mahalonobis distance between the track of the $p$ th target at previous time step, $\boldsymbol{x}_{s_{p}, k}:=\mathbf{F} \boldsymbol{x}_{s_{p, k-1}}$ and the output of the SMCPHD filter, $\tilde{\boldsymbol{x}}_{p, k} ; d_{\max }$ is a constant and it specifies the boundary for the Mahalanobis distance and $-d_{\max } \leq d_{p, k} \leq d_{\max }, \mu_{1}>0$ and $\mu_{2}>0$ are constants and

$$
\mathbf{F}=\left[\begin{array}{llll}
1 & 1 & 0 & 0 \\
0 & 1 & 0 & 0 \\
0 & 0 & 1 & 1 \\
0 & 0 & 0 & 1
\end{array}\right]
$$

. The function $g_{p, k}\left(s_{p, k}, s_{-p, k}\right)$ is defined as

$$
g_{p, k}\left(s_{p, k}, s_{-p, k}\right)= \begin{cases}\frac{1}{\mathcal{P}_{k}-1} \sum_{\substack{l=1 \\ l \neq p}}^{\mathcal{P}_{k}}\left\|s_{p, k}-s_{l}\right\|_{l_{0}}, & T_{k}>1 \\ 1, & T_{k}=1\end{cases}
$$

\section{Equilibrium Points: Correlated Equilibria}

In order to find the equilibrium of the MTT data association game for varying number of targets, correlated equilibrium (CE) [21] as considered in [9] is used. $\mathrm{CE}$ is a generalization of the Nash Equilibrium, (NE). A probability distribution $\varphi$ is called a CE if for all players $p \in \Omega$ and all strategies $i, j \in \mathcal{S}_{p}$, we have [22]

$$
\sum_{s \in \mathcal{S}: s_{p}=i} \varphi(s)\left[u_{p}\left(j, s_{-p}\right)-u_{p}(s)\right] \leq 0 .
$$

A CE, $\varphi$ can be considered as a recommendation each player receives 'privately' from a trusted source. If this source draws a strategy profile $s$ from $\varphi$ and announces to each player $p$ its own component separately and privately, then the player $p$ will have no incentive to choose another strategy, assuming that the other players also conform to the recommendation provided by the source [22]. The CE for the MTT data association game for varying number of targets can be reached using a learning mechanism called regret matching.

\section{Regret Matching with Forgetting Factor}

Regret matching $(\mathrm{RM})$ is a type of learning algorithm used in fully distributed learning [18]. Let $s_{p, k}$ denote the strategy of the $p$ th player in the $k$ th iteration. Note that $s_{p, k} \in\left\{0,1,2, \cdots, T_{k}\right\}$, where $T_{k}$ is the number of strategies at time $k$. Each player computes the average regret for choosing the $n$th strategy for $n \in\left\{0,1,2, \cdots, T_{k}\right\}$ in the $k$ th iteration

$$
\begin{aligned}
r_{p, n}(k) & =\max \left\{0, R_{p, n}(k)\right\} \\
R_{p, n}(k) & =\frac{1}{k-1} \sum_{l=1}^{k-1}\left[u_{p, k}\left(n, s_{-p, k}(l)\right)-u_{p, k}(s(l))\right]
\end{aligned}
$$

Each player $p_{k}$ can recursively compute the $n$th component of $R_{p, k}$ using the recursion:

$$
\begin{aligned}
R_{p, n}(k)= & \left(\frac{k-2}{k-1}\right) R_{p, n}(k-1) \\
& +\frac{1}{k-1}\left[\left(n, s_{-p, k}(k-1)\right)-u_{p, k}(s(k-1))\right]
\end{aligned}
$$

Since the number of players changes with time, the regret in the distant past becomes irrelevant. As a result, we introduce an exponential forgetting factor, $\lambda_{f}$ in (10) to obtain the regret matching with forgetting factor (RMFF) equation:

$$
\tilde{R}_{p, n}(k)=\frac{1}{k-1} \sum_{l=1}^{k-1} \lambda_{f}^{(k-1)-l}\left[u_{p, k}\left(n, s_{-p, k}(l)\right)-u_{p, k}(s(l))\right]
$$

and (9) becomes:

$$
\tilde{r}_{p, n}(k)=\max \left\{0, \tilde{R}_{p, n}(k)\right\}
$$

where $0<\lambda_{f} \leq 1$ is the forgetting factor. The memory of the RMFF equation is given as $\Lambda=\frac{1}{1-\lambda_{f}}$. The expression $\tilde{r}_{p, n}(k)$ in (13) has an interpretation as the measure of the average regret at $k$ th iteration for not having played the strategy $n$ up to time $k$. Let $\beta_{p, n}(k)$ denote the probability that the $p_{k}$ th player chooses $n$th strategy. In regret matching, each player chooses a strategy according to the distribution $\beta_{p}(k)$ which is proportional to the regret vector of the player. When a new player joins the game, i.e. $T_{k}>T_{k-1}$, its own distribution, $\beta_{p}(k)$ is started at that instance. Let $s_{p}(k-1)=\left(l, s_{-p}(k-1)\right), l \in$ $\left\{1,2, \cdots, T_{k}\right\}$. Then

$$
\beta_{p, n}(k)= \begin{cases}\frac{1}{\alpha} \tilde{r}_{p, n}(k), & \text { if } l \neq n \\ 1-\sum_{\substack{n \in\left\{0,1, \ldots, T_{k}\right\} \\ n \neq l}} \beta_{p, n}(k), & l=n\end{cases}
$$

The constant $\alpha>0$ is a large enough number fixed throughout the procedure [18], [23]. This choice of $\alpha$ guarantees that there is always a positive probability of playing the same strategy as in the previous step. It can be seen that in regret matching, the correlation in the plays of different players arises from the commonly observed history. Thus, the history serves as a signal in giving the private recommendation to each player [18].

\section{NUMERICAL RESULTS}

In this section, the performance of the proposed technique is demonstrated. We consider tracking and associating the target state estimates of four targets. These targets enter and exit the tracking scene at various times. Fig. 2 shows the $\mathrm{x}$ and $\mathrm{y}$ components of each track against time. A triangular and circular dot denote the start and the end of a track respectively. To evaluate performance, we
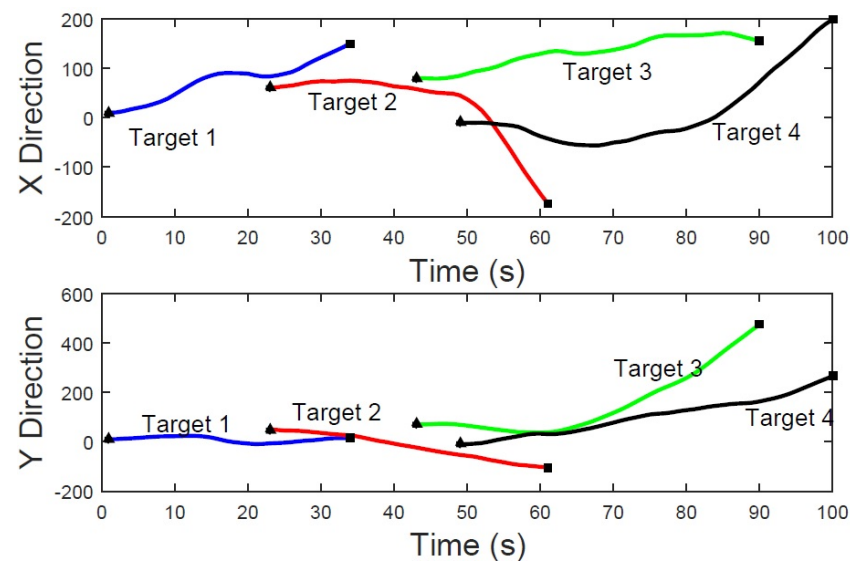

Figure 2: Ground truth showing the plot of the true $x$ and $y$ components against time for the four tracks over 100 time steps

use the root mean squared error (RMSE), track continuity [24] and computational time (CT). The RMSE is computed individually for 
each target as

$$
\operatorname{RMSE}_{t}=\sqrt{\frac{1}{K} \sum_{i=1}^{K}\left(\boldsymbol{x}_{t}^{i}-\tilde{\boldsymbol{x}}_{t}^{i}\right)^{2}}
$$

where $t$ denotes the $t$ th target, $K$ is the number of SMC-PHD filter iterations which is same as the time upto now; $\left\{\boldsymbol{x}_{t}^{i}\right\}_{i=1}^{K}$ denotes the set of ground truth of the $t$ th target and $\left\{\tilde{\boldsymbol{x}}_{t}^{i}\right\}_{i=1}^{K}$ denotes the set of the $t$ th target state estimates after game theoretic data association.

Table I: The performance of the proposed algorithm in terms of RMSE, track continuity and computation time (CT).

\begin{tabular}{|c|ccccc|c|}
\hline \multirow{2}{*}{ Algorithm } & \multicolumn{4}{|c|}{ Track continuity (\%) / RMSE } & \multirow{2}{*}{ CT (s) } \\
\cline { 2 - 5 } & Target 1 & Target 2 & Target 3 & Target 4 & \\
\hline PDA & $94.3 / 3.2$ & $93.9 / 2.9$ & $96.1 / 2.2$ & $96.5 / 2.7$ & 25.0 \\
\hline JPDA & $95.3 / 1.1$ & $94.4 / 1.1$ & $96.7 / 0.9$ & $96.0 / 1.0$ & 22.4 \\
\hline GTDA & $97.1 / 0.7$ & $98.0 / 0.9$ & $98.2 / 1.0$ & $98.8 / 0.8$ & 19.6 \\
\hline
\end{tabular}

Table I show results averaged over 50 Monte Carlo simulations for different data association algorithms with 1000 number of particles used for the SMC-PHD filter. PDA is the probabilistic data association technique [25], JPDA denote joint-PDA [7] and GTDA is our proposed method. Comparing the three algorithms, the GTDA gave the best performance both in terms of accuracy and CT. The JPDA gave a similar level of performance in accuracy when compared with the GTDA but has higher computational time. This is because in JPDA many hypothesis are considered and the hypotheses are merged to form a single one after considering all targets and measurements. The GTDA gave a lower CT and only half of the regrets of the players were kept $\left(\lambda_{f}=0.5\right)$.

\section{CONCLUSION}

A data association technique for MTT with varying number of targets using game theory has been proposed. The strategies and the utility functions of the players are defined, and a regret matching with forgetting factor is used to find the equilibrium of the game. The performance of our technique is compared to other data association algorithms such as the PDA and JPDA. The proposed method shows better performance in terms of accuracy and complexity when compared to PDA. However, our approach showed similar performance in terms of accuracy when compared with JPDA but takes less computational time.

\section{ACKNOWLEDGEMENT}

This work was supported by the Engineering and Physical Sciences Research Council (EPSRC) Grant number EP/K014307/1 and the MOD University Defence Research Collaboration (UDRC) in Signal Processing.

\section{REFERENCES}

[1] R. Sithiravel, X. Chen, M. McDonald, and T. Kirubarajan, "Spline probability hypothesis density filter for nonlinear maneuvering target tracking," in Signals, Systems and Computers, 2013 Asilomar Conference on, 2013, pp. 1743-1450.

[2] Y. Bar-Shalom, P. Willett, P. Willett, and X. Tian, Tracking and Data Fusion: A Handbook of Algorithms. YBS Publishing, 2011. [Online]. Available: http://books.google.co.uk/books?id=2aOiuAAACAAJ

[3] Y. Bar-Shalom, X. R. Li, and T. Kirubarajan, Estimation with Applications to Tracking and Navigation: Theory Algorithms and Software. John Wiley \& Sons, 2004.

[4] Y. Bar-Shalom, P. K. Willett, and X. Tian, "Tracking and data fusion," A Handbook of Algorithms., 2011.

[5] R. P. Mahler, Statistical multisource-multitarget information fusion. Artech House, Inc., 2007.

[6] R. Mahler, "Statistics 102 for multisource-multitarget detection and tracking," Selected Topics in Signal Processing, IEEE Journal of, vol. 7, no. 3, pp. 376-389, 2013.
[7] T. E. Fortmann, Y. Bar-Shalom, and M. Scheffe, "Sonar tracking of multiple targets using joint probabilistic data association," Oceanic Engineering, IEEE Journal of, vol. 8, no. 3, pp. 173-184, Jul 1983.

[8] P. Chavali and A. Nehorai, "Concurrent particle filtering and data association using game theory for tracking multiple maneuvering targets," Signal Processing, IEEE Transactions on, vol. 61, no. 20, pp. 4934-4948, 2013.

[9] - "Distributed data association for multiple-target tracking using game theory," in Radar Conference (RADAR), 2013 IEEE, April 2013, pp. 1-6.

[10] B. Jia, M. Xin, and Y. Cheng, "Sparse-grid quadrature nonlinear filtering," Automatica, vol. 48, no. 2, pp. 327-341, 2012.

[11] A. Smith, A. Doucet, N. de Freitas, and N. Gordon, Sequential Monte Carlo methods in practice. Springer Science \& Business Media, 2013.

[12] R. L. Streit, Poisson Point Processes: Imaging, Tracking, and Sensing. Springer Science \& Business Media, 2010.

[13] B.-N. Vo and W.-K. Ma, "The Gaussian mixture probability hypothesis density filter," Signal Processing, IEEE Transactions on, vol. 54, no. 11, pp. 4091-4104, 2006.

[14] R. Sithiravel, X. Chen, R. Tharmarasa, B. Balaji, and T. Kirubarajan, "The spline probability hypothesis density filter," Signal Processing, IEEE Transactions on, vol. 61, no. 24, pp. 6188-6203, 2013.

[15] B.-T. Vo, B.-N. Vo, and A. Cantoni, "The cardinality balanced multitarget multi-Bernoulli filter and its implementations," Signal Processing, IEEE Transactions on, vol. 57, no. 2, pp. 409-423, 2009.

[16] — "The cardinality balanced multi-target multi-Bernoulli filter and its implementations," Signal Processing, IEEE Transactions on, vol. 57, no. 2, pp. 409-423, 2009.

[17] B.-N. Vo, S. Singh, and A. Doucet, "Random finite sets and sequential Monte Carlo methods in multi-target tracking," in Radar Conference, 2003. Proceedings of the International, 2003, pp. 486-491.

[18] S. Hart and A. Mas-Colell, "A simple adaptive procedure leading to correlated equilibrium1," Econometrica, vol. 68, no. 5, p. 11271150, 2000.

[19] B.-N. Vo, S. Singh, and A. Doucet, "Sequential Monte Carlo implementation of the PHD filter for multi-target tracking," in Information Fusion, 2003. Proceedings of the Sixth International Conference of, vol. 2, 2003, pp. 792-799.

[20] _ "Sequential Monte Carlo methods for multitarget filtering with random finite sets," Aerospace and Electronic Systems, IEEE Transactions on, vol. 41, no. 4, pp. 1224-1245, 2005.

[21] R. J. Aumann, "Subjectivity and correlation in randomized strategies," Journal of mathematical Economics, vol. 1, no. 1, pp. 67-96, 1974.

[22] S. Hart and A. Mas-Colell, "A simple adaptive procedure leading to correlated equilibrium1," Econometrica, vol. 68, no. 5, p. 11271150, 2000.

[23] S. Lasaulce and H. Tembine, Game theory and learning for wireless networks: fundamentals and applications. Academic Press, 2011.

[24] C. Coman and T. Kreitmair, "Evaluation of the tracking process in ground surveillance applications," in Radar Conference, 2009. EuRAD 2009. European, 2009, pp. 553-556.

[25] Y. Bar-Shalom and E. Tse, "Tracking in a cluttered environment with probabilistic data association," Automatica, vol. 11, no. 5, pp. 451-460, 1975. 